\title{
Depiction of peak to average power ratio reduction scheme and potentials for $5 \mathrm{G}$
}

\begin{abstract}
Peak to Average Power Ratio (PAR or PAPR) is one of the most challenging issues in the operation of Orthogonal Frequency Division Multiplexing (OFDM) for multicarrier signals used in Fourth and Fifth Generation of broadband cellular network technology (4G and 5G). There are numerous PAPR reduction or also recognized as Crest Factor Reduction (CFR) techniques, for instance Clipping, Coding, Dummy Sequence Insertion (DSI), Tone Reservation, Active Constellation Sequence (ACE), Partial Transmit Sequence (PTS), and Selective Mapping (SLM) schemes. Among these methods, SLM-based techniques are very attractive solutions due to their good performance without additional-of-band radiations or inband distortions. This study demonstrates a performance analysis of an SLM-based method combined with adding randomly generated dummy sequences to power-free subcarriers. Simulation results show that the PAPR of the OFDM based signal can be reduced efficiently by using adequate number of dummies and tolerable number of iterations, and that is a potential scheme for $4 \mathrm{G}$ and $5 \mathrm{G}$.
\end{abstract}

Keyword: CCDF; CFR; Dummy sequence insertion; OFDM; PAPR; SLM; 5G 